Lef schet z i nvar i ant s and Young char acters for repr esent at i ons of the hyper oct ahedral groups

\begin{tabular}{|l|l|}
\hline 著者 & $\begin{array}{l}\text { ODA Fumi hi to, TAKEGAHARA Yugen, YOSH DA } \\
\text { Tonoy uki }\end{array}$ \\
\hline $\begin{array}{l}\mathrm{j} \text { our nal or } \\
\text { publ i cat } \mathrm{i} \text { on } \mathrm{ti} \mathrm{tl} \text { e }\end{array}$ & Jour nal of Al gebr a \\
\hline vol une & 512 \\
\hline page $\mathrm{r}$ ange & $1-19$ \\
\hline year & $2018-10$ 15 \\
\hline URL & ht $\mathrm{p}: / /$ hdl . handl e. net /10258/00009673 \\
\hline
\end{tabular}




\title{
Lefschetz invariants and Young characters for representations of the hyperoctahedral groups
}

\author{
Fumihito Oda \\ Department of Mathematics, Kindai University, Higashi-Osaka, 577-8502, Japan \\ E-mail: odaf@math.kindai.ac.jp \\ Yugen Takegahara* \\ Muroran Institute of Technology, 27-1 Mizumoto, Muroran 050-8585, Japan \\ E-mail: yugen@mmm.muroran-it.ac.jp

\section{Tomoyuki Yoshida} \\ Graduate School of Economics, Hokusei Gakuen University, 2-3-1, Ohyachi-Nishi, \\ Atsubetsu-ku, Sapporo 004-8631, Japan \\ E-mail: ytomoyuki@mub.biglobe.ne.jp
}

\begin{abstract}
The ring $R\left(B_{n}\right)$ of virtual $\mathbb{C}$-characters of the hyperoctahedral group $B_{n}$ has two $\mathbb{Z}$-bases consisting of permutation characters, and the ring structure associated with each basis of them defines a partial Burnside ring of which $R\left(B_{n}\right)$ is a homomorphic image. In particular, the concept of Young characters of $B_{n}$ arises from a certain set $\mathcal{U}_{n}$ of subgroups of $B_{n}$, and the $\mathbb{Z}$-basis of $R\left(B_{n}\right)$ consisting of Young characters, which is presented by L. Geissinger and D. Kinch [7], forces $R\left(B_{n}\right)$ to be isomorphic to a partial Burnside ring $\Omega\left(B_{n}, \mathcal{U}_{n}\right)$. The linear $\mathbb{C}$-characters of $B_{n}$ are analyzed with reduced Lefschetz invariants which characterize the unit group of $\Omega\left(B_{n}, \mathcal{U}_{n}\right)$. The parabolic Burnside ring $\mathcal{P B}\left(B_{n}\right)$ is a subring of $\Omega\left(B_{n}, \mathcal{U}_{n}\right)$, and the unit group of $\mathcal{P} \mathcal{B}\left(B_{n}\right)$ is isomorphic to the four group. The unit group of the parabolic Burnside ring of the evensigned permutation group $D_{n}$ is also isomorphic to the four group.
\end{abstract}

${ }^{*}$ This work was supported by JSPS KAKENHI Grant Number JP16K05052.

2010 Mathematics Subject Classification. Primary 19A22; Secondary 20B30, 20B35, 20C15, 20C30.

Keywords. Burnside ring, Character ring, Hyper octahedral group, Lefschetz invariant, Parabolic subgroup, Sign character, Symmetric group, Young subgroup. 


\section{Introduction}

Let $G$ be a finite group, and let $G$-set be the category of finite left $G$-sets and $G$-equivariant maps. The Burnside ring $\Omega(G)$, which is the Grothendieck ring of the category $G$-set, is the commutative unital ring consisting of all $\mathbb{Z}$-linear combinations of isomorphism classes $[X]$ of finite left $G$-sets $X$ with disjoint union for addition and cartesian product for multiplication. We denote by $R(G)$ the ring of virtual $\mathbb{C}$-characters of $G$. Set $[n]=\{1,2, \ldots, n\}$, and let $S_{n}$ be the symmetric group on $[n]$. We denote by $\mathcal{Y}_{n}$ the set of Young subgroups of $S_{n}$, which is closed under intersection and conjugation. By $[15, \S 7], \Omega\left(S_{n}\right)$ possesses the partial Burnside ring $\Omega\left(S_{n}, \mathcal{Y}_{n}\right)$ relative to the Young subgroups as a subring, and $\Omega\left(S_{n}, \mathcal{Y}_{n}\right) \cong R\left(S_{n}\right)$. This fact means that the characters $1_{Y}^{S_{n}}$ induced from the trivial characters $1_{Y}$ of $Y$ for $Y \in \mathcal{Y}_{n}$ form a $\mathbb{Z}$-basis of $R\left(S_{n}\right)$ (see, e.g., [2, Proposition 3]). Let $C_{2}$ be a cyclic group of order 2 , and let $V_{n}$ be the direct product $C_{2}^{(n)}$ of $n$ copies of $C_{2}$. We denote by $B_{n}$ the hyperoctahedral group, that is, the wreath product $C_{2} 2 S_{n}$ defined to be a semidirect product $V_{n} \rtimes S_{n}$ of $V_{n}$ with $S_{n}$. Let $\mathcal{Z}_{n}$ be the set of all products $K Y$ of $K \leq V_{n}$ and $Y \in \mathcal{Y}_{n}$ with $\left|V_{n}: K\right| \leq 2$ and $Y \leq N_{S_{n}}(K)$. We establish in $\S 3$ that $R\left(B_{n}\right)$ is a homomorphic image of the partial Burnside ring $\Omega\left(B_{n}, \widetilde{\mathcal{Z}}_{n}\right)$ relative to the set $\widetilde{\mathcal{Z}}_{n}$ of intersections of subgroups contained in $\mathcal{Z}_{n}$.

For a ring $R$, we denote by $R^{\times}$the unit group of $R$. By [13, Example 2], $R\left(S_{n}\right)^{\times}$ is isomorphic to the four group. There exists a unit of $\Omega\left(S_{n}, \mathcal{Y}_{n}\right)$ which enables us to describe the sign character $\operatorname{sgn}_{n}: S_{n} \rightarrow \mathbb{C}$ as a $\mathbb{Z}$-linear combination of the characters $1_{Y}^{S_{n}}$ for $Y \in \mathcal{Y}_{n}$ (see [2, Corollary 2] and [9, §4]); such a description is called Solomon's formula. The ring $R\left(B_{n}\right)$ includes exactly four linear $\mathbb{C}$-characters, and $R\left(B_{n}\right)^{\times}$is generated by the nontrivial linear $\mathbb{C}$-characters and $-1_{B_{n}}$. In $\S 4$ we identify $R\left(B_{n}\right)^{\times}$with a subgroup of $\Omega\left(B_{n}, \widetilde{\mathcal{Z}}_{n}\right)^{\times}$, and then describe the linear $\mathbb{C}$-characters of $B_{n}$ as $\mathbb{Z}$-linear combinations of the characters $1_{H}^{B_{n}}$ for $H \in \mathcal{Z}_{n}$.

There is a set $\mathcal{U}_{n}$ of subgroups of $B_{n}$ such that the characters $1_{H}^{B_{n}}$ for $H \in \mathcal{U}_{n}$ form a $\mathbb{Z}$-basis of $R\left(B_{n}\right)$ (cf. [7, Corollary II.4]). In $\S 5$ we define the partial Burnside ring $\Omega\left(B_{n}, \mathcal{U}_{n}\right)$ relative to the Young subgroups of $B_{n}$, which is a subring of $\Omega\left(B_{n}\right)$ isomorphic to $R\left(B_{n}\right)$. The parabolic Burnside $\operatorname{ring} \mathcal{P} \mathcal{B}\left(B_{n}\right)$ (cf. [1, §4]) is a subring of $\Omega\left(B_{n}, \mathcal{U}_{n}\right)$. By $\left[4,(66.29)\right.$ Corollary], the sign character $\varepsilon_{n}: B_{n} \rightarrow \mathbb{C}$ is described as a $\mathbb{Z}$-linear combination of the characters $1_{H}^{B_{n}}$ for parabolic subgroups $H$ of $B_{n}$, whence $\mathcal{P B}\left(B_{n}\right)$ includes a unit $\alpha_{n}$ corresponding to $\varepsilon_{n}: B_{n} \rightarrow \mathbb{C}$. There also is a unit $\beta_{n}$ of $\Omega\left(B_{n}, \mathcal{U}_{n}\right)$ corresponding to a natural extension of $\operatorname{sgn}_{n}: S_{n} \rightarrow \mathbb{C}$ to $B_{n}$ such that $\alpha_{n} \beta_{n}$ corresponds to the restriction of $\operatorname{sgn}_{2 n}: S_{2 n} \rightarrow \mathbb{C}$ to $B_{n}$. By the description of $\beta_{n}$ in terms of the characters $1_{H}^{B_{n}}$ for $H \in \mathcal{Z}_{n} \cap \mathcal{U}_{n}$, we have

$$
\beta_{n} \in \Omega\left(B_{n}, \widetilde{\mathcal{Z}}_{n}\right)^{\times} \cap\left(\Omega\left(B_{n}, \mathcal{U}_{n}\right)^{\times}-\mathcal{P} \mathcal{B}\left(B_{n}\right)^{\times}\right),
$$

which proves $\mathcal{P} \mathcal{B}\left(B_{n}\right)^{\times}$to be isomorphic to the four group.

Let $X \in G$-set. To explore the units of $\Omega(G)$, we are mainly concerned with the reduced Lefschetz invariant $\widetilde{\Lambda}_{P(X)}$ of the $G$-poset $P(X)$ consisting of nonempty 
and proper subsets of $X$. The reduced Euler-Poincaré characteristic $\widetilde{\chi}\left(P(X)^{K}\right)$ of the set of $K$-invariants $P(X)^{K}$ in $P(X)$ with $K \leq G$ is $(-1)^{|K \backslash X|}$, so that $\widetilde{\Lambda}_{P(X)}$ is a unit of $\Omega(G)$ (cf. $[11, \S 5]$ ). As a sequel to this fact, the linear $\mathbb{C}$-characters of $B_{n}$ are analyzed with reduced Lefschetz invariants which characterize $\Omega\left(B_{n}, \mathcal{U}_{n}\right)^{\times}$.

Let $D_{n}$ be the group of even-signed permutations on $[n]$, which is also a Coxeter group of type $D$. In $\S 6$ we explore the units of the parabolic Burnside ring of $D_{n}$.

\section{Lefschetz invariant}

Following $[4, \S 80]$, we review the Burnside ring of $G$ and related facts. Let $\mathbf{F}(G)$ be the free abelian group on the set of isomorphism classes of finite left $G$-sets. Given $X \in G$-set, we denote by $\bar{X}$ the isomorphism class of left $G$-sets including $X$. Let $\mathbf{F}(G)_{0}$ be the subgroup of $\mathbf{F}(G)$ generated by the elements $\overline{X_{1} \cup X_{2}}-\overline{X_{1}}-\overline{X_{2}}$ for $X_{1}, X_{2} \in G$-set. We define a multiplication on the generators of $\mathbf{F}(G)$ by

$$
\overline{X_{1}} \cdot \overline{X_{2}}=\overline{X_{1} \times X_{2}},
$$

where $X_{1} \times X_{2}$ is the cartesian product of $X_{1}$ and $X_{2}$, and extend it to $\mathbf{F}(G)$ by $\mathbb{Z}$-linearly. Then $\mathbf{F}(G)$ is a commutative unital ring, and $\mathbf{F}(G)_{0}$ is an ideal of $\mathbf{F}(G)$. We define a commutative unital ring $\Omega(G)$ to be the quotient $\mathbf{F}(G) / \mathbf{F}(G)_{0}$, and call it the Burnside ring of $G$. For each $X \in G$-set, let $[X]$ be the coset $\bar{X}+\mathbf{F}(G)_{0}$ of $\mathbf{F}(G)_{0}$ in $\mathbf{F}(G)$ represented by $\bar{X}$. Then by [4, (80.4) Lemma], $\left[X_{1}\right]=\left[X_{2}\right]$ if and only if $\overline{X_{1}}=\overline{X_{2}}$. Hence we may regard $[X]$ as the isomorphism class of left $G$-sets including $X \in G$-set. Multiplication on the generators of $\Omega(G)$ is given by

$$
\left[X_{1}\right] \cdot\left[X_{2}\right]=\left[X_{1} \times X_{2}\right] .
$$

Let $\mathrm{C}(G)$ be a full set of non-conjugate subgroups of $G$. Given $H \leq G$, we denote by $G / H$ the set of left cosets $g H, g \in G$, of $H$ in $G$, and make $G / H$ into a left $G$-set by defining $d(g H)=d g H$ for all $d, g \in G$. For $H, K \leq G, G / H \simeq G / K$ if and only if $H$ is a conjugate of $K$ (cf. [4, (80.5) Proposition]). The elements $[G / H]$ for $H \in \mathrm{C}(G)$ form a free $\mathbb{Z}$-basis of $\Omega(G)$. We have

$$
[G / H] \cdot[G / U]=\sum_{H g U \in H \backslash G / U}\left[G /\left(H \cap{ }^{g} U\right)\right]
$$

for all $H, U \leq G$, where ${ }^{g} U=g U g^{-1}$ (cf. [4, $\S 80$ Exercise 2]). The identity of $\Omega(G)$ is $[G / G]$. For shortness' sake, we usually write $1=[G / G]$.

Let $H \leq G$. For each $X \in G$-set, we denote by $\operatorname{inv}_{H}(X)$ or $X^{H}$ the set of $H$-invariants in $X$. There exists a ring homomorphism $\phi_{H}: \Omega(G) \rightarrow \mathbb{Z}$ given by

$$
[G / U] \mapsto\left|\operatorname{inv}_{H}(G / U)\right|
$$

for all $U \in \mathrm{C}(G)$. For each $X \in G$-set, it is obvious that

$$
\phi_{H}([X])=\left|X^{H}\right| \text {. }
$$


We set $\widetilde{\Omega}(G)=\prod_{H \in \mathrm{C}(G)} \mathbb{Z}$, and define a map $\phi: \Omega(G) \rightarrow \widetilde{\Omega}(G)$ by

$$
x \mapsto\left(\phi_{H}(x)\right)_{H \in \mathrm{C}(G)}
$$

for all $x \in \Omega(G)$. By $[4,(80.12)$ Proposition], this map is a ring monomorphism. We call $\widetilde{\Omega}(G)$ the ghost ring of $\Omega(G)$, and call $\phi: \Omega(G) \rightarrow \widetilde{\Omega}(G)$ the Burnside homomorphism or the mark homomorphism. Obviously, $\widetilde{\Omega}(G)^{\times}=\prod_{H \in \mathrm{C}(G)} \mathbb{Z}^{\times}$. Hence $\widetilde{\Omega}(G)^{\times}$is an elementary abelian 2-group, and so is $\Omega(G)^{\times}$.

We turn to the concept of (reduced) Lefschetz invariants for finite $G$-sets. A finite (left) $G$-set $P$ equipped with order relation $\leq$ is called a finite $G$-poset if $\leq$ is invariant under the $G$-action. Let $P$ be a finite $G$-poset. For each nonnegative integer $n$, we denote by $S d_{n}(P)$ the set of chains $p_{0}<p_{1}<\cdots<p_{n}$ of elements of $P$ of cardinality $n+1$, and make $S d_{n}(P)$ into a $G$-set by defining

$$
g\left(p_{0}<p_{1}<\cdots<p_{n}\right)=g p_{0}<g p_{1}<\cdots<g p_{n}
$$

for all $g \in G$ and $p_{0}<p_{1}<\cdots<p_{n} \in S d_{n}(P)$. The Lefschetz invariant $\Lambda_{P}$ of $P$ and the reduced Lefschetz invariant $\widetilde{\Lambda}_{P}$ of $P$ are two elements of $\Omega(G)$ given by

$$
\Lambda_{P}=\sum_{i=0}^{\infty}(-1)^{i}\left[S d_{i}(P)\right] \text { and } \widetilde{\Lambda}_{P}=\Lambda_{P}-1,
$$

respectively, which are introduced by Thévenaz (cf. $[3,11])$.

Given $X \in G$-set, we denote by $P(X)$ the $G$-poset consisting of nonempty and proper subsets of $X$, and explore $\widetilde{\Lambda}_{P(X)}$ from the point of view of combinatorics.

Definition 2.1 Let $X \in G$-set. Given $X_{0} \in G$-set, we define a finite left $G$-set $\operatorname{Map}\left(X, X_{0}\right)$ to be the set of maps from $X$ to $X_{0}$ with the action given by

$$
(g f)(x)=g f\left(g^{-1} x\right)
$$

for all $g \in G, f \in \operatorname{Map}\left(X, X_{0}\right)$, and $x \in X$ (cf. [5, §2]). Given a nonnegative integer $i$ and $X_{0}, X_{1}, \ldots, X_{i} \in G$-set, we denote by $\operatorname{Map}\left(X, X_{0}, X_{1}, \ldots, X_{i}\right)$ the set of all $f \in \operatorname{Map}\left(X, X_{0} \dot{\cup} X_{1} \dot{\cup} \cdots \dot{\cup} X_{i}\right)$ such that $\operatorname{Im} f \cap X_{j} \neq \emptyset$ for any $j=1,2, \ldots, i$, and make it into a left $G$-set by defining

$$
(g f)(x)=g f\left(g^{-1} x\right)
$$

for all $g \in G, f \in \operatorname{Map}\left(X, X_{0}, X_{1}, \ldots, X_{i}\right)$, and $x \in X$.

Lemma 2.2 Let $X \in G$-set. Set $n=|X|$ and $X_{1}=\cdots=X_{n}=G / G$. Then

$$
\widetilde{\Lambda}_{P(X)}=\sum_{i=1}^{n}(-1)^{i}\left[\operatorname{Map}\left(X, \emptyset, X_{1}, \ldots, X_{i}\right)\right] .
$$


Proof. Obviously, $\left[\operatorname{Map}\left(X, \emptyset, X_{1}\right)\right]=[\operatorname{Map}(X, G / G)]=1$. We assume that $2 \leq i \leq$ $n$, and define a bijection $\Delta: \operatorname{Map}\left(X, \emptyset, X_{1}, \ldots, X_{i}\right) \rightarrow S d_{i-2}(P(X))$ by

$$
f \mapsto p_{0}<p_{1}<\cdots<p_{i-2}
$$

where

$$
p_{k}=\left\{x \in X \mid f(x) \in X_{j} \text { for some } j \in\{1,2, \ldots, k+1\}\right\}
$$

for each integer $k$ with $0 \leq k \leq i-2$. Let $g \in G$, and let $f \in \operatorname{Map}\left(X, \emptyset, X_{1}, \ldots, X_{i}\right)$. We have $(g f)(g x)=f(x)$ for any $x \in X$. Hence, if $\Delta(f)=p_{0}<p_{1}<\cdots<p_{i-2}$, then $\Delta(g f)=g p_{0}<g p_{1}<\cdots<g p_{i-2}$. Consequently, we have

$$
\left[\operatorname{Map}\left(X, \emptyset, X_{1}\right)\right]=1 \quad \text { and } \quad\left[\operatorname{Map}\left(X, \emptyset, X_{1}, \ldots, X_{i}\right)\right]=\left[S d_{i-2}(P(X))\right]
$$

for all integer $i$ with $2 \leq i \leq n$, which implies that

$$
\widetilde{\Lambda}_{P(X)}=-1+\sum_{i=0}^{\infty}(-1)^{i}\left[S d_{i}(P(X))\right]=\sum_{i=1}^{n}(-1)^{i}\left[\operatorname{Map}\left(X, \emptyset, X_{1}, \ldots, X_{i}\right)\right] .
$$

This completes the proof.

By Eq.(1), the set $\Omega(G)^{+}$consisting of all elements $\sum_{U \in \mathrm{C}(G)} \ell_{U}[G / U], \ell_{U} \geq 0$, of $\Omega(G)$ is an additive semigroup closed under multiplication. We fix $X \in G$-set, and define a multiplicative map $\operatorname{Map}(X,-): \Omega(G)^{+} \rightarrow \Omega(G)$ by

$$
[Y] \mapsto[\operatorname{Map}(X, Y)]
$$

for all $Y \in G$-set. There exists a unique polynomial map (multiplicative map) $(-)^{[X]}: \Omega(G) \rightarrow \Omega(G), y \mapsto y^{[X]}$ extending $\operatorname{Map}(X,-)$ (see $[5, \S 2]$ and $[14, \S 3]$ ). If $X=X_{1} \dot{\cup} X_{2}$, then $y^{[X]}=y^{\left[X_{1}\right]} \cdot y^{\left[X_{2}\right]}$ for any $y \in \Omega(G)$.

By [14, Lemma 3.6], $\phi\left((-1)^{[X]}\right)=\left((-1)^{|K \backslash X|}\right)_{K \in \mathrm{C}(G)}$, where $K \backslash X$ is the set of $K$-orbits in $X$, and thus $(-1)^{[X]} \in \Omega(G)^{\times}$. The following proposition is equivalent to $[9$, Proposition 4.1] and [11, Proposition 5.1].

Proposition 2.3 For any $X \in G$-set, $\widetilde{\Lambda}_{P(X)}=(-1)^{[X]} \in \Omega(G)^{\times}$.

We derive Proposition 2.3 from the combinatorial identity

$$
(-1)^{n}=\sum_{i=1}^{n}(-1)^{i} S(n, i) i !
$$

where $S(n, i)$ is the Stirling number of the second kind (cf. [10, (24d)]). While Eq.(2) is equivalent to [9, Lemma 4.2], the former is nicer than the later for our argument based on entry 3 of the Twelvefold Way (cf. [10, p. 33]). 
Proof of Proposition 2.3. Set $n=|X|$ and $X_{1}=\cdots=X_{n}=G / G$. By Lemma 2.2,

$$
\widetilde{\Lambda}_{P(X)}=\sum_{i=1}^{n}(-1)^{i}\left[\operatorname{Map}\left(X, \emptyset, X_{1}, \ldots, X_{i}\right)\right] .
$$

Let $K \in \mathrm{C}(G)$, and set $m_{K}=|K \backslash X|$. Then for each integer $i$ with $1 \leq i \leq n$,

$$
\left|\operatorname{Map}\left(X, \emptyset, X_{1}, \ldots, X_{i}\right)^{K}\right|=S\left(m_{K}, i\right) i !
$$

because $S\left(m_{K}, i\right)$ is the number of partitions of an $m_{K}$-set into $i$ nonempty subsets. Combining the preceding facts with Eq.(2), we have

$$
\phi\left(\widetilde{\Lambda}_{P(X)}\right)=\left(\sum_{i=1}^{m_{K}}(-1)^{i} S\left(m_{K}, i\right) i !\right)_{K \in \mathrm{C}(G)}=\left((-1)^{m_{K}}\right)_{K \in \mathrm{C}(G)},
$$

completing the proof.

Remark 2.4 For each $X \in G$-set, the elements $y^{[X]}$ for $y \in \Omega(G)$, which may be called exponentials, were introduced by A. Dress (cf. [5, §2]), including $(-1)^{[X]}$ (cf. $[5, \S 3])$, and the fact that $\phi\left(\widetilde{\Lambda}_{P(X)}\right)=\left((-1)^{|K \backslash X|}\right)_{K \in \mathrm{C}(G)}$ was generalized in terms of the exponentials (see $[12, \S 6]$ and $[14, \S 3])$.

\section{The character ring of $B_{n}$}

Set $C_{2}=\mathbb{Z}^{\times}$, and let $V_{n}$ be the direct product $C_{2}^{(n)}$ of $n$ copies of $C_{2}$. The wreath product $B_{n}:=C_{2}$ 乙 $S_{n}$ of $C_{2}$ with $S_{n}$ is defined to be the semidirect product

$$
V_{n} \rtimes S_{n}=\left\{\left(x_{1}, \ldots, x_{n}\right) \sigma \mid\left(x_{1}, \ldots, x_{n}\right) \in V_{n} \text { and } \sigma \in S_{n}\right\}
$$

in which each permutation on $[n]$ acts as an inner automorphism on $V_{n}$ :

$$
\sigma\left(x_{1}, \ldots, x_{n}\right) \sigma^{-1}=\left(x_{\sigma^{-1}(1)}, \ldots, x_{\sigma^{-1}(n)}\right) .
$$

If $L \leq V_{n}$ or if $F \leq S_{n}$, then we regard $L$ or $F$ as a subgroup of $B_{n}$. Given $K \leq V_{n}$ and $F \leq N_{S_{n}}(K):=N_{B_{n}}(K) \cap S_{n}, K F$ is the semidirect product $K \rtimes F$.

Given $J \subset[n]$, we denote by $S_{J}$ the symmetric group on $J$, and view it as a subgroup of $S_{n}$. For a cycle type $\lambda=\left(1^{m_{1}}, \ldots, n^{m_{n}}\right)$ of a permutation on $[n]$, let $S_{\lambda}$ denote a Young subgroup of $S_{n}$ isomorphic to $S_{1}^{\left(m_{1}\right)} \times \cdots \times S_{n}^{\left(m_{n}\right)}$, where each $S_{i}^{\left(m_{i}\right)}$ is the direct product of $m_{i}$ copies of $S_{i}$.

Let $J \subset[n]$. There exists a linear $\mathbb{C}$-character $\vartheta_{J}$ of $V_{n}$ given by

$$
\vartheta_{J}\left(\left(x_{1}, \ldots, x_{n}\right)\right)=\vartheta\left(x_{1}\right) \cdots \vartheta\left(x_{n}\right) \quad \text { with } \quad \vartheta\left(x_{j}\right)=\left\{\begin{array}{cl}
x_{j} & \text { if } j \in J, \\
1 & \text { otherwise }
\end{array}\right.
$$


for all $\left(x_{1}, \ldots, x_{n}\right) \in V_{n}$. Set $\bar{J}=[n]-J$. The inertia group $I_{B_{n}}\left(\vartheta_{J}\right)$ of $\vartheta_{J}$, which is defined to be $\left\{a \in B_{n} \mid \vartheta_{J}\left(a b a^{-1}\right)=\vartheta_{J}(b)\right.$ for all $\left.b \in V_{n}\right\}$, is

$$
V_{n}\left(S_{J} S_{\bar{J}}\right)=\left\{\left(x_{1}, \ldots, x_{n}\right) \sigma \in B_{n} \mid\left(x_{1}, \ldots, x_{n}\right) \in V_{n} \text { and } \sigma \in S_{J} S_{\bar{J}}\right\}
$$

(cf. [8, Lemma 25.5]). There exists an extension $\widehat{\vartheta_{J}}$ of $\vartheta_{J}$ to $I_{B_{n}}\left(\vartheta_{J}\right)$ given by

$$
\widehat{\vartheta_{J}}\left(\left(x_{1}, \ldots, x_{n}\right) \sigma\right)=\vartheta_{J}\left(\left(x_{1}, \ldots, x_{n}\right)\right)
$$

for all $\left(x_{1}, \ldots, x_{n}\right) \in V_{n}$ and $\sigma \in S_{J} S_{\bar{J}}$. Obviously, $I_{B_{n}}\left(\vartheta_{J}\right) / V_{n} \simeq S_{J} S_{\bar{J}}$. For a $\mathbb{C}$-character $\psi$ of $S_{J} S_{\bar{J}}$, we denote by $\widehat{\psi}$ the $\mathbb{C}$-character of $I_{B_{n}}\left(\vartheta_{J}\right)$ given by

$$
\widehat{\psi}(g \sigma)=\psi(\sigma)
$$

for all $g \in V_{n}$ and $\sigma \in S_{J} S_{\bar{J}}$. Set $K_{J}=\operatorname{ker} \vartheta_{J}$. Then $S_{J} S_{\bar{J}} \leq I_{B_{n}}\left(\vartheta_{J}\right) \leq N_{B_{n}}\left(K_{J}\right)$.

For each integer $i$ with $0 \leq i \leq n$, we indicate with $[i] \subset[n]$ that $[i]$ is the subset $\{1,2, \ldots, i\}$ of $[n]$, where $[0]$ is the empty set.

Let $[i] \subset[n]$. We write $\vartheta_{i}=\vartheta_{[i]}, K_{i}=\operatorname{ker} \vartheta_{i}, S_{i}=S_{[i]}$, and $S_{\bar{i}}=S_{\overline{[i]}}$ for shortness' sake. Let $\operatorname{Irr}\left(S_{i} S_{\bar{i}}\right)$ be the set of irreducible $\mathbb{C}$-characters of $S_{i} S_{\bar{i}}$.

The following proposition is well-known (cf. $[7, \S \mathrm{II}])$.

Proposition 3.1 The irreducible $\mathbb{C}$-characters of $B_{n}$ consist of the $\mathbb{C}$-characters $\left(\widehat{\vartheta}_{i} \widehat{\psi}\right)^{B_{n}}$ induced from the product $\widehat{\vartheta}_{i} \widehat{\psi}$ of $\widehat{\vartheta}_{i}$ and $\widehat{\psi}$ for $[i] \subset[n]$ and $\psi \in \operatorname{Irr}\left(S_{i} S_{\bar{i}}\right)$.

Let $J \subset[n]$, and let $\mathcal{P}(J)$ be the set of cycle types of permutations on $J$. We write $\mathcal{P}(n)=\mathcal{P}([n])$. Recall that for each $\lambda \in \mathcal{P}(J)(=\mathcal{P}(|J|)), S_{\lambda}$ denotes a Young subgroup of $S_{|J|}$. We set $\mathcal{P}(J, \bar{J})=\mathcal{P}(J) \times \mathcal{P}(\bar{J})$. Given $\left(\lambda_{J}, \lambda_{\bar{J}}\right) \in \mathcal{P}(J, \bar{J})$, let $S_{\lambda_{J} \lambda_{\bar{J}}}$ denote the product $H K$ of a subgroup $H$ of $S_{J}$ and a subgroup $K$ of $S_{\bar{J}}$ such that $H$ is a conjugate of $S_{\lambda_{J}}$ in $S_{n}$ and $K$ is a conjugate of $S_{\lambda_{J}}$ in $S_{n}$.

For each $X \in G$-set, let $\pi_{X}$ be the permutation character of $G$ which assigns each $g \in G$ the number of fixed elements of $X$ by $g$, that is, $\pi_{X}(g)=\left|X^{\langle g\rangle}\right|$. For each $H \leq G, \pi_{G / H}$ is the character $1_{H}^{G}$ induced from the trivial character $1_{H}$ of $H$.

Theorem 3.2 The characters $1_{K_{i} S_{\lambda_{i} \lambda_{\bar{i}}}}^{B_{n}}$ induced from the trivial characters $1_{K_{i} S_{\lambda_{i} \lambda_{\bar{i}}}}$ of $K_{i} S_{\lambda_{i} \lambda_{\bar{i}}}$ for $[i] \subset[n]$ and $\left(\lambda_{i}, \lambda_{\bar{i}}\right) \in \mathcal{P}([i], \overline{[i]})$ form a $\mathbb{Z}$-basis of $R\left(B_{n}\right)$. In particular, the number of irreducible $\mathbb{C}$-characters of $B_{n}$ is $\sum_{i=0}^{n}|\mathcal{P}([i], \overline{[i]})|$.

Proof. The second assertion is well-known, and is also an immediate consequence of the first one. Let $J \subset[n]$, and let $\left(\lambda_{J}, \lambda_{\bar{J}}\right) \in \mathcal{P}(J, \bar{J})$. If $g \in V_{n}$ and $\sigma \in S_{J} S_{\bar{J}}$, then

$$
\begin{aligned}
g \sigma\left(h \tau K_{J} S_{\lambda_{J} \lambda_{\bar{J}}}\right)=h \tau K_{J} S_{\lambda_{J} \lambda_{\bar{J}}} & \Longleftrightarrow \tau^{-1} h^{-1}(g \sigma) h \tau \in K_{J} S_{\lambda_{J} \lambda_{\bar{J}}} \\
& \Longleftrightarrow \tau^{-1}\left(h^{-1} g\right)^{-1} \sigma h \tau^{-1} \sigma \tau \in K_{J} S_{\lambda_{J} \lambda_{\bar{J}}} \\
& \Longleftrightarrow g{ }^{\sigma} h h^{\tau} K_{J} \text { and } \sigma \tau \in \tau S_{\lambda_{J} \lambda_{\bar{J}}} \\
& \Longleftrightarrow g h K_{J}=h K_{J} \text { and } \sigma \tau S_{\lambda_{J} \lambda_{\bar{J}}}=\tau S_{\lambda_{J} \lambda_{\bar{J}}}
\end{aligned}
$$


for all $h \in V_{n}$ and $\tau \in S_{J} S_{\bar{J}}$, because $\sigma \in N_{S_{n}}\left(K_{J}\right)$ and $\left|V_{n}: K_{J}\right| \leq 2$, and thus

$$
\begin{aligned}
1_{K_{J} S_{\lambda_{J} \lambda_{\bar{J}}}}^{I_{B_{J}}\left(\vartheta_{J}\right)}(g \sigma) & =\pi_{I_{B_{n}}\left(\vartheta_{J}\right) /\left(K_{J} S_{\lambda_{J} \lambda_{\bar{J}}}\right)}(g \sigma) \\
& =\pi_{V_{n} / K_{J}}(g) \cdot \pi_{\left(S_{J} S_{\bar{J}}\right) / S_{\lambda_{J} \lambda_{\bar{J}}}}(\sigma) \\
& =1_{K_{J}}^{V_{n}}(g) \cdot 1_{S_{\lambda_{J} \lambda_{\bar{J}}}}^{S_{S_{\bar{J}}}}(\sigma) .
\end{aligned}
$$

In particular, $1_{V_{n} S_{\lambda_{\bar{\emptyset}}}}^{I_{B_{n}}\left(\vartheta_{\emptyset}\right)}=1_{S_{\lambda_{\bar{\emptyset}}}}^{\widehat{S_{\bar{\emptyset}}}}$. Moreover, if $J \neq \emptyset$, then $\vartheta_{J}=1_{K_{J}}^{V_{n}}-1_{V_{n}}$ and

$$
\left(1_{K_{J} S_{\lambda_{J} \lambda_{\bar{J}}}}^{I_{B_{n}}\left(\vartheta_{J}\right)}-\widehat{1_{S_{\lambda_{J} \lambda_{\bar{J}}} S_{\overline{\bar{J}}}}}\right)(g \sigma)=\left(1_{K_{J}}^{V_{n}}-1_{V_{n}}\right)(g) \cdot 1_{S_{\lambda_{J} \lambda_{\bar{J}}}}^{S_{J} S_{\overline{\bar{J}}}}(\sigma)=\left(\widehat{\vartheta_{J}} 1_{S_{\lambda_{J} \lambda_{\bar{J}}}}^{S_{\bar{J}}}\right)(g \sigma)
$$

for all $g \in V_{n}$ and $\sigma \in S_{J} S_{\bar{J}}$, and consequently,

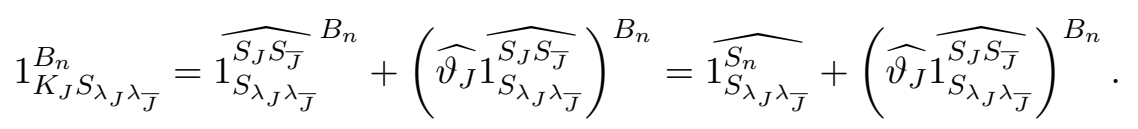

Let $[i] \subset[n]$. By the above fact with $J=[i]$ and Proposition 3.1, it suffices to verify that the characters $1_{S_{\lambda_{i} \lambda_{\bar{i}}}}^{S_{i} S_{\overline{\bar{l}}}}$ for $\left(\lambda_{i}, \lambda_{\bar{i}}\right) \in \mathcal{P}([i], \overline{[i]})$ form a $\mathbb{Z}$-basis of $R\left(S_{i} S_{\bar{i}}\right)$. We identify $S_{i} S_{\bar{i}}$ and the subgroups $S_{\lambda_{i} \lambda_{\bar{i}}}$ of $S_{i} S_{\bar{i}}$ for $\left.\left(\lambda_{i}, \lambda_{\bar{i}}\right) \in \mathcal{P}([i], \bar{i}]\right)$ with $S_{i} \times S_{n-i}$ and the subgroups $S_{\mu} \times S_{\nu}$ of $S_{i} \times S_{n-i}$ for $\mu \in \mathcal{P}(i)$ and $\nu \in \mathcal{P}(n-i)$, respectively. By [2, Proposition 3] and [4, §9 Exercise 6], the characters $1_{S_{\mu} \times S_{n-i}}^{S_{i} \times S_{n-i}} 1_{S_{i} \times S_{\nu}}^{S_{i} \times S_{n-i}}$ for $\mu \in \mathcal{P}(i)$ and $\nu \in \mathcal{P}(n-i)$ form a $\mathbb{Z}$-basis of $R\left(S_{i} \times S_{n-i}\right)$. This, combined with [4, (10.19) Corollary], shows that the characters $1_{S_{\mu} \times S_{\nu}}^{S_{i} \times S_{n-i}}$ for $\mu \in \mathcal{P}(i)$ and $\nu \in \mathcal{P}(n-i)$ form a $\mathbb{Z}$-basis of $R\left(S_{i} \times S_{n-i}\right)$, as desired. This completes the proof.

We quote part of $[15, \S 3]$ and review the concept of generalized Burnside rings.

Definition 3.3 For a set $\mathcal{D}$ of subgroups of $G$, we define a $\mathbb{Z}$-lattice $\Omega(G, \mathcal{D})$ to be an additive group consisting of all $\mathbb{Z}$-linear combinations of the elements $[G / H]$ of $\Omega(G)$ for $H \in \mathcal{D}$, and define $\overline{\mathcal{D}}:=\left\{{ }^{g} H \mid g \in G\right.$ and $\left.H \in \mathcal{D}\right\}$.

The following theorem is a concise version of [15, 3.11 Theorem].

Theorem 3.4 Let $\mathcal{D}$ be a set of subgroups of $G$ including $G$, and suppose that

$$
\bigcap_{\langle g\rangle U \leq H \in \overline{\mathcal{D}}} H \in \overline{\mathcal{D}}
$$

for all $U \in \overline{\mathcal{D}}$ and $g \in N_{G}(U)$. Then $\Omega(G, \overline{\mathcal{D}})$ has a unique ring structure such that the group homomorphism $\Omega(G, \overline{\mathcal{D}}) \rightarrow \prod_{H \in \mathrm{C}(G) \cap \overline{\mathcal{D}}} \mathbb{Z}$ given by

$$
x \mapsto\left(\phi_{H}(x)\right)_{H \in \mathrm{C}(G) \cap \overline{\mathcal{D}}}
$$

for all $x \in \Omega(G, \overline{\mathcal{D}})$ is a ring homomorphism, and the identity of $\Omega(G, \overline{\mathcal{D}})$ is 1 . If $\overline{\mathcal{D}}$ is closed under intersection, then $\Omega(G, \overline{\mathcal{D}})$ is a subring of $\Omega(G)$. 
We set $\mathcal{X}_{n}=\left\{K_{J} S_{\lambda_{J} \lambda_{\bar{J}}} \mid J \subset[n]\right.$ and $\left.\left(\lambda_{J}, \lambda_{\bar{J}}\right) \in \mathcal{P}(J, \bar{J})\right\}$. Let $\mathcal{Y}_{n}$ be the set of Young subgroups of $S_{n}$, and let $\mathcal{Z}_{n}$ be the set consisting of all products $K Y$ of $K \leq V_{n}$ and $Y \in \mathcal{Y}_{n}$ with $\left|V_{n}: K\right| \leq 2$ and $Y \leq N_{S_{n}}(K)$. We define

$$
\widetilde{\mathcal{Z}}_{n}:=\left\{\bigcap_{H \in \mathcal{S}} H \mid \mathcal{S} \in \operatorname{Sub}\left(\mathcal{Z}_{n}\right)\right\},
$$

where $\operatorname{Sub}\left(\mathcal{Z}_{n}\right)$ is the set of nonempty subsets of $\mathcal{Z}_{n}$.

Lemma 3.5 The following statements hold.

(a) The set $\overline{\mathcal{X}_{n}}$ coincides with $\mathcal{Z}_{n}$. In particular, $\mathcal{Z}_{n}$ is closed under conjugation.

(b) The set $\widetilde{\mathcal{Z}}_{n}$ is closed under intersection and conjugation.

Proof. Suppose that $J \subset[n]$ and $\left(\lambda_{J}, \lambda_{\bar{J}}\right) \in \mathcal{P}(J, \bar{J})$. Let $\sigma \in S_{n}$, and let $g \in V_{n}$. Then we have ${ }^{\sigma}\left(K_{J} S_{\lambda_{J} \lambda_{\bar{J}}}\right)=K_{\sigma(J)}{ }^{\sigma} S_{\lambda_{J} \lambda_{\bar{J}}},{ }^{\sigma} S_{\lambda_{J} \lambda_{\bar{J}}} \in \mathcal{Y}_{n}$, and ${ }^{\sigma} S_{\lambda_{J} \lambda_{\bar{J}}} \leq N_{S_{n}}\left(K_{\sigma(J)}\right)$, where $\sigma(J)=\{\sigma(j) \mid j \in J\}$. Since $\vartheta_{J}\left(g^{\tau} g\right)=1$ for any $\tau \in S_{J} S_{\bar{J}}$, it follows that

$$
{ }^{g}\left(K_{J} S_{\lambda_{J} \lambda_{\bar{J}}}\right)=\left\{g h^{\tau} g \tau \mid h \in K_{J} \text { and } \tau \in S_{\lambda_{J} \lambda_{\bar{J}}}\right\}=K_{J} S_{\lambda_{J} \lambda_{\bar{J}}} .
$$

In particular, $\overline{\mathcal{X}_{n}} \subset \mathcal{Z}_{n}$. Suppose that $K \leq V_{n}$ and $Y \in \mathcal{Y}_{n}$ with $\left|V_{n}: K\right| \leq 2$ and $Y \leq N_{S_{n}}(K)$. There exists a subset $J$ of $[n]$ such that $K=K_{J}$. For each $\sigma \in Y$, we have $K_{J}={ }^{\sigma}\left(K_{J}\right)=K_{\sigma(J)}$, whence $\sigma(J)=J$ and $Y={ }^{\tau} S_{\lambda_{J} \lambda_{\bar{J}}}$ for some $\tau \in S_{J} S_{\bar{J}}$ and $\left(\lambda_{J}, \lambda_{\bar{J}}\right) \in \mathcal{P}(J, \bar{J})$. This means that $K Y$ is a conjugate of $K_{J} S_{\lambda_{J} \lambda_{\bar{J}}}$. Consequently, $\overline{\mathcal{X}_{n}} \supset \mathcal{Z}_{n}$, and the statement (a) holds. Obviously, $\widetilde{\mathcal{Z}}_{n}$ is closed under intersection. Hence the statement (b) follows from (a). This completes the proof.

By Lemma 3.5, $\widetilde{\mathcal{Z}}_{n}$ satisfies the hypothesis of Theorem 3.4 with $\mathcal{D}=\overline{\mathcal{D}}=\widetilde{\mathcal{Z}}_{n}$, so that $\Omega\left(B_{n}, \widetilde{\mathcal{Z}}_{n}\right)$ is a subring of $\Omega\left(B_{n}\right)$ which is called a partial Burnside ring.

We now define a ring homomorphism $\operatorname{char}_{G}: \Omega(G) \rightarrow R(G)$ by

$$
[X] \mapsto \pi_{X}
$$

for all $X \in G$-set (cf. $[14, \S 6]$ ), and usually write char $=\operatorname{char}_{G}$ by omitting subscript $G$. Given $x \in \Omega(G)$ and $g \in G, \operatorname{char}(x)(g)=\phi_{\langle g\rangle}(x)$.

We are successful in finding a natural relationship between $\Omega\left(B_{n}, \widetilde{\mathcal{Z}}_{n}\right)$ and $R\left(B_{n}\right)$.

Theorem 3.6 The ring homomorphism char: $\Omega\left(B_{n}\right) \rightarrow R\left(B_{n}\right)$ induces an epimorphism from the partial Burnside ring $\Omega\left(B_{n}, \widetilde{\mathcal{Z}}_{n}\right)$ to $R\left(B_{n}\right)$.

Proof. The theorem is a consequence of Theorem 3.2. 


\section{Units of the character ring of $B_{n}$}

The set $[n]$ is viewed as a left $S_{n}$-set. According to [9, Eq.(3)],

$$
\widetilde{\Lambda}_{P([n])}=\sum_{\lambda=\left(1^{m_{1}}, \ldots, n^{m_{n}}\right) \in \mathcal{P}(n)}(-1)^{m_{1}+\cdots+m_{n}} \frac{\left(m_{1}+\cdots+m_{n}\right) !}{m_{1} ! \cdots m_{n} !}\left[S_{n} / S_{\lambda}\right],
$$

so that the sign character $\operatorname{sgn}_{n}: S_{n} \rightarrow \mathbb{C}$ is described as

$$
\operatorname{sgn}_{n}=\sum_{\lambda=\left(1^{m_{1}}, \ldots, n^{m_{n}}\right) \in \mathcal{P}(n)}(-1)^{m_{1}+\cdots+m_{n}+n} \frac{\left(m_{1}+\cdots+m_{n}\right) !}{m_{1} ! \cdots m_{n} !} 1_{S_{\lambda}}^{S_{n}}
$$

(see [2, Corollary 2] and [9, Theorem 4.4]). Note that the numbers

$$
\frac{\left(m_{1}+\cdots+m_{n}\right) !}{m_{1} ! \cdots m_{n} !}
$$

for nonnegative integers $m_{1}, \ldots, m_{n}$ are multinomial coefficients (cf. [10, 1.2]).

Let $\kappa_{n}: B_{n} \rightarrow \mathbb{C}$ be a linear $\mathbb{C}$-character of $B_{n}$ given by

$$
\left(x_{1}, \ldots, x_{n}\right) \sigma \mapsto \prod_{i=1}^{n} x_{i}
$$

for all $\left(x_{1}, \ldots, x_{n}\right) \in V_{n}$ and $\sigma \in S_{n}$. There also exists an extension $\rho_{n}: B_{n} \rightarrow \mathbb{C}$ of the sign character $\operatorname{sgn}_{n}: S_{n} \rightarrow \mathbb{C}$ to $B_{n}$ given by

$$
\left(x_{1}, \ldots, x_{n}\right) \sigma \mapsto \operatorname{sgn}_{n}(\sigma)
$$

for all $\left(x_{1}, \ldots, x_{n}\right) \in V_{n}$ and $\sigma \in S_{n}$. Let $\varepsilon_{n}: B_{n} \rightarrow \mathbb{C}$ be the product $\kappa_{n} \rho_{n}$ of $\kappa_{n}$ and $\rho_{n}$, which coincides with the sign character of $B_{n}$.

We view the set $\mathbb{Z}^{\times}=\{1,-1\}$ as a left $B_{n^{-}}$set with the action given by

$$
\left(x_{1}, \ldots, x_{n}\right) \sigma \cdot x=x \cdot \prod_{i=1}^{n} x_{i}
$$

for all $\left(x_{1}, \ldots, x_{n}\right) \in V_{n}, \sigma \in S_{n}$, and $x \in \mathbb{Z}^{\times}$. The set $[n]$ is naturally viewed as a left $B_{n}$-set on which $V_{n}$ acts trivially. Let $[n]^{\diamond}$ denote the $B_{n}$-set $\mathbb{Z}^{\times} \dot{\cup}[n]$.

Lemma 4.1 There are exactly three nontrivial linear $\mathbb{C}$-characters $\kappa_{n}: B_{n} \rightarrow \mathbb{C}$, $\rho_{n}: B_{n} \rightarrow \mathbb{C}$, and $\varepsilon_{n}: B_{n} \rightarrow \mathbb{C}$ defined as above in $R\left(B_{n}\right)$, and $\kappa_{n}(y)=(-1)^{\left|\langle y\rangle \backslash \mathbb{Z}^{\times}\right|}$, $\rho_{n}(y)=(-1)^{|\langle y\rangle \backslash[n]|+n}$, and $\varepsilon_{n}(y)=(-1)^{\left|\langle y\rangle \backslash[n]^{\diamond}\right|+n}$ for each $y \in B_{n}$.

Proof. By Proposition 3.1, there are exactly three nontrivial linear $\mathbb{C}$-characters of $B_{n}$. Let $\left(x_{1}, \ldots, x_{n}\right) \in V_{n}$, and let $\sigma \in S_{n}$. Set $y=\left(x_{1}, \ldots, x_{n}\right) \sigma \in B_{n}$, and 
assume that $\sigma$ is a product of pairwise disjoint $n_{j}$-cycles $\sigma_{j}$ for $j=1,2, \ldots, r$ with $\sum_{j} n_{j}=n$. Obviously, $\kappa_{n}(y)=(-1)^{\left|\langle y\rangle \backslash \mathbb{Z}^{\times}\right|}$. We have $|\langle y\rangle \backslash[n]|=r$ and

$$
\left|\langle y\rangle \backslash[n]^{\diamond}\right|= \begin{cases}r+1 & \text { if } \prod_{i=1}^{n} x_{i}=-1 \\ r+2 & \text { if } \prod_{i=1}^{n} x_{i}=1 .\end{cases}
$$

Moreover, if $\ell=\sharp\left\{j \mid n_{j}\right.$ is odd $\}$, then $\rho_{n}(y)=\operatorname{sgn}(\sigma)=(-1)^{r-\ell}=(-1)^{r+n}$ and $\varepsilon_{n}(y)=(-1)^{r+n} \prod_{i=1}^{n} x_{i}$, because $\ell \equiv n(\bmod 2)$. This completes the proof.

Lemma $4.2 R\left(B_{n}\right)^{\times}=\left\langle\kappa_{n}, \eta_{n},-1_{B_{n}}\right\rangle$.

Proof. The lemma is a consequence of [6, Theorem 5.5.6] (see also Theorem 3.2), [13, Corollary 1.2 and Lemma 2.1], and Lemma 4.1.

We are now in position to establish the following proposition.

Proposition 4.3 The nontrivial linear $\mathbb{C}$-characters of $B_{n}$ are characterized by the reduced Lefschetz invariants. Indeed, $\kappa_{n}=\operatorname{char}\left(\widetilde{\Lambda}_{P\left(\mathbb{Z}^{\times}\right)}\right), \rho_{n}=(-1)^{n} \operatorname{char}\left(\widetilde{\Lambda}_{P([n])}\right)$, and $\varepsilon_{n}=(-1)^{n} \operatorname{char}\left(\widetilde{\Lambda}_{P\left([n]^{\diamond}\right)}\right)$. The reduced Lefschetz invariants $\widetilde{\Lambda}_{P\left(\mathbb{Z}^{\times}\right)}$and $\widetilde{\Lambda}_{P([n])}$, together with -1 , generate an elementary abelian subgroup of $\Omega\left(B_{n}, \widetilde{\mathcal{Z}}_{n}\right)^{\times}$isomorphic to $R\left(B_{n}\right)^{\times}$, and $\widetilde{\Lambda}_{P\left([n]^{\diamond}\right)}=\widetilde{\Lambda}_{P([n])} \cdot \widetilde{\Lambda}_{P\left(\mathbb{Z}^{\times}\right)}$. Moreover,

$$
\begin{aligned}
& \widetilde{\Lambda}_{P\left(\mathbb{Z}^{\times}\right)}=\left[B_{n} /\left(K_{n} S_{n}\right)\right]-\left[B_{n} / B_{n}\right], \\
& \widetilde{\Lambda}_{P([n])}=\sum_{\lambda=\left(1^{m_{1}}, \ldots, n^{m_{n}}\right) \in \mathcal{P}(n)}(-1)^{m_{1}+\cdots+m_{n}} \frac{\left(m_{1}+\cdots+m_{n}\right) !}{m_{1} ! \cdots m_{n} !}\left[B_{n} /\left(V_{n} S_{\lambda}\right)\right], \\
& \widetilde{\Lambda}_{P\left([n]^{\triangleleft}\right)}=\sum_{\lambda=\left(1^{m_{1}}, \ldots, n^{m_{n}}\right) \in \mathcal{P}(n)}(-1)^{m_{1}+\cdots+m_{n}} \frac{\left(m_{1}+\cdots+m_{n}\right) !}{m_{1} ! \cdots m_{n} !}\left[B_{n} /\left(K_{n} S_{\lambda}\right)\right] \\
& -\sum_{\lambda=\left(1^{m_{1}}, \ldots, n^{m_{n}}\right) \in \mathcal{P}(n)}(-1)^{m_{1}+\cdots+m_{n}} \frac{\left(m_{1}+\cdots+m_{n}\right) !}{m_{1} ! \cdots m_{n} !}\left[B_{n} /\left(V_{n} S_{\lambda}\right)\right] .
\end{aligned}
$$

Proof. The first assertion follows from Proposition 2.3 and Lemma 4.1. We prove the last two assertions. By Lemma 2.2 with $X=\mathbb{Z}^{\times}$and $X_{1}=X_{2}=B_{n} / B_{n}$,

$$
\widetilde{\Lambda}_{P\left(\mathbb{Z}^{\times}\right)}=-\left[\operatorname{Map}\left(\mathbb{Z}^{\times}, \emptyset, X_{1}\right)\right]+\left[\operatorname{Map}\left(\mathbb{Z}^{\times}, \emptyset, X_{1}, X_{2}\right)\right]=-\left[B_{n} / B_{n}\right]+\left[B_{n} /\left(K_{n} S_{n}\right)\right] .
$$

We obtain the description of $\widetilde{\Lambda}_{P([n])}$ in a similar fashion to the proof of [9, Eq.(3)]. By Proposition 2.3, $\widetilde{\Lambda}_{P\left([n]^{\diamond}\right)}=\widetilde{\Lambda}_{P([n])} \cdot \widetilde{\Lambda}_{P\left(\mathbb{Z}^{\times}\right)}$, which yields the description of $\widetilde{\Lambda}_{P\left([n]^{\diamond}\right)}$, and the reduced Lefschetz invariants $\widetilde{\Lambda}_{P\left(\mathbb{Z}^{\times}\right)}, \widetilde{\Lambda}_{P([n])}$, and $\widetilde{\Lambda}_{P\left([n]^{\diamond}\right)}$ are contained 
in $\Omega\left(B_{n}, \widetilde{\mathcal{Z}}_{n}\right)^{\times}$. Hence it follows from Lemma 4.2 that $\widetilde{\Lambda}_{P\left(\mathbb{Z}^{\times}\right)}, \widetilde{\Lambda}_{P([n])}$, and -1 generate an elementary abelian subgroup of $\Omega\left(B_{n}, \widetilde{\mathcal{Z}}_{n}\right)^{\times}$isomorphic to $R\left(B_{n}\right)^{\times}$. This completes the proof.

The following descriptions of nontrivial linear $\mathbb{C}$-characters of $B_{n}$ are obtained; see Eq.(5) in $\S 5$ for Solomon's formula of the sign character $\varepsilon_{n}: B_{n} \rightarrow \mathbb{C}$.

\section{Corollary 4.4}

$$
\begin{aligned}
\kappa_{n} & =1_{K_{n} S_{n}}^{B_{n}}-1_{B_{n}}, \\
\rho_{n} & =\sum_{\lambda=\left(1^{m_{1}}, \ldots, n^{m_{n}}\right) \in \mathcal{P}(n)}(-1)^{m_{1}+\cdots+m_{n}+n} \frac{\left(m_{1}+\cdots+m_{n}\right) !}{m_{1} ! \cdots m_{n} !} 1_{V_{n} S_{\lambda}}^{B_{n}}, \\
\varepsilon_{n} & =\sum_{\lambda=\left(1^{m_{1}}, \ldots, n^{m_{n}}\right) \in \mathcal{P}(n)}(-1)^{m_{1}+\cdots+m_{n}+n} \frac{\left(m_{1}+\cdots+m_{n}\right) !}{m_{1} ! \cdots m_{n} !} 1_{K_{n} S_{\lambda}}^{B_{n}} \\
& \quad-\sum_{\lambda=\left(1^{m_{1}}, \ldots, n^{m_{n}}\right) \in \mathcal{P}(n)}(-1)^{m_{1}+\cdots+m_{n}+n} \frac{\left(m_{1}+\cdots+m_{n}\right) !}{m_{1} ! \cdots m_{n} !} 1_{V_{n} S_{\lambda}}^{B_{n}} .
\end{aligned}
$$

Proof. The corollary is an immediate consequence of Proposition 4.3. (The formulae of $\kappa_{n}$ and $\rho_{n}$ can also be obtained by a calculation and Eq.(4), respectively.)

\section{The Young subgroups of the hyperoctahedral groups}

Given $J \subset[n]$, we define a subgroup $L_{J}$ of $V_{n}$ by

$$
L_{J}=\left\{\left(x_{1}, \ldots, x_{n}\right) \in V_{n} \mid x_{k}=1 \text { for all } k \in \bar{J}\right\} .
$$

Let $\mathcal{U}_{n}$ denote the set of products $L_{J} S_{\lambda_{J} \lambda_{\bar{J}}}$ of $L_{J}$ and $S_{\lambda_{J} \lambda_{\bar{J}}}$ for $J \subset[n]$ and $\left(\lambda_{J}, \lambda_{\bar{J}}\right) \in \mathcal{P}(J, \bar{J})$, and let $\mathcal{E}_{n}$ denote the set of products $L_{J}\left(S_{\lambda_{J} \lambda_{\bar{J}}} S_{J}\right)$ of $L_{J}$ and $S_{\lambda_{J} \lambda_{\bar{J}}} S_{J}$ for $J \subset[n]$ and $\left(\lambda_{J}, \lambda_{\bar{J}}\right) \in \mathcal{P}(J, \bar{J})$. Obviously, $\mathcal{E}_{n} \subset \mathcal{U}_{n}$.

We call the subgroups $L_{J} S_{\lambda_{J} \lambda_{\bar{J}}}$ of $B_{n}$ and the characters $1_{L_{J} S_{\lambda_{J} \lambda_{J}}}^{B_{n}}$ for $J \subset[n]$ and $\left(\lambda_{J}, \lambda_{\bar{J}}\right) \in \mathcal{P}(J, \bar{J})$ the Young subgroups and the Young characters, respectively.

The sets $\mathcal{U}_{n}$ and $\mathcal{E}_{n}$ are closed under intersection; they are not closed under conjugation, however. Recall that $\overline{\mathcal{D}}=\left\{{ }^{y} H \mid y \in B_{n}\right.$ and $\left.H \in \mathcal{D}\right\}$ where $\mathcal{D}$ is $\mathcal{U}_{n}$ or $\mathcal{E}_{n}$. Given $[i] \subset[n]$ and $\lambda \in \mathcal{P}(i)$, we write $L_{\bar{i}}=L_{\overline{[i]}}$ and $S_{\lambda} B_{n-i}=L_{\bar{i}}\left(S_{\lambda} S_{\bar{i}}\right)$. The set $\overline{\mathcal{E}}_{n}$ consists of the conjugates of the parabolic subgroups $S_{\lambda} B_{n-i}$ for $[i] \subset[n]$ and $\lambda \in \mathcal{P}(i)$, and is closed under intersection (cf. [6, Exercise 2.2]). To explore $\overline{\mathcal{U}}_{n}$, we make $\mathbb{Z}^{\times} \times[n]$ into a left $B_{n}$-set by defining

$$
\left(x_{1}, x_{2}, \ldots, x_{n}\right) \sigma \cdot(x, i)=\left(x_{\sigma(i)} x, \sigma(i)\right)
$$

for all $\left(x_{1}, x_{2}, \ldots, x_{n}\right) \in V_{n}, \sigma \in S_{n}$, and $(x, i) \in \mathbb{Z}^{\times} \times[n]$. 
Lemma 5.1 The set $\overline{\mathcal{U}}_{n}$ is closed under intersection.

Proof. Suppose that $J_{1}, J_{2} \subset[n],\left(\lambda_{J_{1}}, \lambda_{\overline{J_{1}}}\right) \in \mathcal{P}\left(J_{1}, \overline{J_{1}}\right),\left(\lambda_{J_{2}}, \lambda_{\overline{J_{2}}}\right) \in \mathcal{P}\left(J_{2}, \overline{J_{2}}\right)$, $g \in V_{n}$, and $\sigma \in S_{n}$. Then $g\left(L_{\sigma\left(J_{1}\right)}{ }^{\sigma} S_{\lambda_{J_{1}} \lambda_{\overline{J_{1}}}}\right) \cap L_{J_{2}} S_{\lambda_{J_{2}} \lambda_{\bar{J}}}$ is considered to be the intersection of the stabilizers of disjoint subsets

$$
N_{1}^{+}, \ldots, N_{k}^{+}, N_{1}^{-}, \ldots, N_{k}^{-}, N_{k+1}, \ldots, N_{r}
$$

obtained by a certain partition of $\mathbb{Z}^{\times} \times[n]$ into nonempty subsets such that

$$
N_{i}^{+}=\left\{g_{i} \cdot(1, q) \mid q \in Q_{i}\right\} \quad \text { and } \quad N_{i}^{-}=\left\{g_{i} \cdot(-1, q) \mid q \in Q_{i}\right\}
$$

with $Q_{i} \subset[n]$ and $g_{i} \in L_{Q_{i}}$ for $i=1,2, \ldots, k$ and

$$
N_{i}=\left\{(1, q),(-1, q) \mid q \in Q_{i}\right\}
$$

with $Q_{i} \subset[n]$ for $i=k+1, \ldots, r$. Set $g^{\prime}=g_{1} \cdots g_{k}$ and $J=Q_{k+1} \dot{\cup} \cdots \dot{\cup} Q_{r}$. Then

$$
\begin{aligned}
g \sigma\left(L_{J_{1}} S_{\lambda_{J_{1}} \lambda_{\overline{J_{1}}}}\right) \cap L_{J_{2}} S_{\lambda_{J_{2}} \lambda_{\overline{J_{2}}}} & ={ }^{g}\left(L_{\sigma\left(J_{1}\right)}{ }^{\sigma} S_{\lambda_{J_{1}} \lambda_{\overline{J_{1}}}}\right) \cap L_{J_{2}} S_{\lambda_{J_{2}} \lambda_{\overline{J_{2}}}} \\
& =g^{\prime}\left(L_{J}{ }^{\tau} S_{\lambda_{J} \lambda_{\bar{J}}}\right) \\
& =g^{\prime}{ }^{\prime}\left(L_{J} S_{\lambda_{J} \lambda_{\bar{J}}}\right)
\end{aligned}
$$

for some $\tau \in S_{J} S_{\bar{J}}$ and $\left(\lambda_{J}, \lambda_{\bar{J}}\right) \in \mathcal{P}(J, \bar{J})$. Consequently, $\overline{\mathcal{U}}_{n}$ is closed under intersection. This completes the proof.

By Lemma 5.1 and [6, Exercise 2.2], $\Omega\left(B_{n}, \mathcal{U}_{n}\right)$ and $\Omega\left(B_{n}, \mathcal{E}_{n}\right)$ are subrings of $\Omega\left(B_{n}\right)$ (cf. Theorem 3.4) called partial Burnside rings. The partial Burnside ring $\Omega\left(B_{n}, \mathcal{E}_{n}\right)$ is known as the parabolic Burnside ring. As for the partial Burnside ring $\Omega\left(B_{n}, \mathcal{U}_{n}\right)$ relative to the Young subgroups of $B_{n}$, we quote [7, Corollary II.4]:

Theorem 5.2 The characters $1_{L_{\bar{i}} S_{\lambda_{i} \lambda_{\bar{i}}}}^{B_{n}}$ induced from the trivial characters $1_{L_{\bar{i}} S_{\lambda_{i} \lambda_{\bar{i}}}}$ of $L_{\bar{i}} S_{\lambda_{i} \lambda_{\bar{i}}}$ for $[i] \subset[n]$ and $\left(\lambda_{i}, \lambda_{\bar{i}}\right) \in \mathcal{P}([i], \overline{[i]})$ form a $\mathbb{Z}$-basis of $R\left(B_{n}\right)$.

Corollary 5.3 The ring homomorphism char : $\Omega\left(B_{n}\right) \rightarrow R\left(B_{n}\right)$ induces a ring isomorphism $\overline{\text { char }: ~} \Omega\left(B_{n}, \mathcal{U}_{n}\right) \rightarrow R\left(B_{n}\right)$. In particular, $\Omega\left(B_{n}, \mathcal{U}_{n}\right)^{\times} \simeq R\left(B_{n}\right)^{\times}$.

Proof. The corollary is a consequence of Theorem 5.2, because $\mathcal{U}_{n}$ is a set of conjugates of the subgroups $L_{\bar{i}} S_{\lambda_{i} \lambda_{\bar{i}}}$ for $[i] \subset[n]$ and $\left(\lambda_{i}, \lambda_{\bar{i}}\right) \in \mathcal{P}([i], \overline{[i]})$.

The rest of this section is devoted to quite a new view of the units of $\Omega\left(B_{n}, \mathcal{U}_{n}\right)$.

Proposition 5.4 $\left|\Omega\left(B_{n}, \mathcal{E}_{n}\right)^{\times}\right|=4$. 
Proof. By [4, (66.29) Corollary] and Corollary 5.3, there is a unique unit $\alpha_{n}$ of $\Omega\left(B_{n}, \mathcal{E}_{n}\right)$ such that $\operatorname{char}\left(\alpha_{n}\right)=\varepsilon_{n}$. Obviously, $-1 \in \Omega\left(B_{n}, \mathcal{E}_{n}\right)^{\times}$. Hence we have $\left|\Omega\left(B_{n}, \mathcal{E}_{n}\right)^{\times}\right| \geq 4$. By Proposition 4.3 and Theorem 5.2, $\widetilde{\Lambda}_{P([n])} \in \Omega\left(B_{n}, \mathcal{U}_{n}\right)^{\times}$and $\widetilde{\Lambda}_{P([n])} \notin \Omega\left(B_{n}, \mathcal{E}_{n}\right)^{\times}$. Thus $\left|\Omega\left(B_{n}, \mathcal{U}_{n}\right)^{\times}: \Omega\left(B_{n}, \mathcal{E}_{n}\right)^{\times}\right| \geq 2$. By Lemma 4.1 and Corollary 5.3, we have $\left|\Omega\left(B_{n}, \mathcal{U}_{n}\right)^{\times}\right|=\left|R\left(B_{n}\right)^{\times}\right|=8$, whence $\left|\Omega\left(B_{n}, \mathcal{E}_{n}\right)^{\times}\right|=4$. This completes the proof.

We present a technical lemma by which [4, (66.29) Corollary] deduces Eq.(4) and a description of $\varepsilon_{n}: B_{n} \rightarrow \mathbb{C}$ (see also [6, Propositions 2.3.8 and 2.3.10]):

$$
\varepsilon_{n}=\sum_{i=0}^{n} \sum_{\lambda=\left(1^{m_{1}}, \ldots, i^{m_{i}}\right) \in \mathcal{P}(i)}(-1)^{m_{1}+\cdots+m_{i}+n} \frac{\left(m_{1}+\cdots+m_{i}\right) !}{m_{1} ! \cdots m_{i} !} 1_{S_{\lambda} B_{n-i}}^{B_{n}} .
$$

Lemma 5.5 Let $\left(S_{n}, X\right)$ be the Coxeter system of type $A_{n-1}$. Given $\lambda \in \mathcal{P}(n)$, let $\mathcal{W}(\lambda)$ be the set of parabolic subgroups $W_{I}$ of $S_{n}$ for $I \subset X$ which are conjugates of $S_{\lambda}$. Suppose that $I \subset X$ and $W_{I} \in W(\lambda)$ with $\lambda=\left(1^{m_{1}}, \ldots, n^{m_{n}}\right) \in \mathcal{P}(n)$. Then $|I| \equiv m_{1}+\cdots+m_{n}+n(\bmod 2)$, so that $(-1)^{|I|}=(-1)^{m_{1}+\cdots+m_{n}+n}$.

Proof. We use induction with respect to the partially order $\leq$ on $\mathcal{P}(n)$ given by

$$
\mu \leq \nu \quad: \Longleftrightarrow S_{\mu} \text { is a conjugate of a subgroup of } S_{\nu} .
$$

If $\lambda=\left(1^{n}\right)$, then $I=\emptyset$, and hence $|I| \equiv 2 n(\bmod 2)$. Assume that $\left(1^{n}\right)<\lambda$. Then $m_{k} \neq 0$ and $m_{k+1}=\cdots=m_{n}=0$ for some $k \in[n]$. We set

$$
\mu= \begin{cases}\left(1^{m_{1}+2}, 2^{m_{2}-1}\right) & \text { if } k=2 \\ \left(1^{m_{1}+1}, 2^{m_{2}}, \ldots,(k-1)^{m_{k-1}+1}, k^{m_{k}-1}, 0, \ldots, 0\right) & \text { if } k>2\end{cases}
$$

Suppose that $I^{\prime} \subset X$ and $W_{I^{\prime}} \in W(\mu)$. Then $\mu<\lambda$ and $\left|I^{\prime}\right|=|I|-1$. By the inductive assumption, $\left|I^{\prime}\right| \equiv m_{1}+\cdots+m_{n}+1+n(\bmod 2)$. Since $|I|=\left|I^{\prime}\right|+1$, it follows that $|I| \equiv m_{1}+\cdots+m_{n}+n(\bmod 2)$. This completes the proof.

What about a unique unit $\gamma_{n}$ of $\Omega\left(B_{n}, \mathcal{U}_{n}\right)$ satisfying $\operatorname{char}\left(\gamma_{n}\right)=\kappa_{n}$ ? We are interested in the reduced Lefschetz invariant $\widetilde{\Lambda}_{P\left(\mathbb{Z}^{\times} \times[n]\right)}$.

Lemma $5.6 \kappa_{n}=\operatorname{char}\left(\widetilde{\Lambda}_{P\left(\mathbb{Z}^{\times} \times[n]\right)}\right)$.

Proof. By Proposition 2.3, $\operatorname{char}\left(\widetilde{\Lambda}_{P\left(\mathbb{Z}^{\times} \times[n]\right)}\right)(y)=(-1)^{\left|\langle y\rangle \backslash\left(\mathbb{Z}^{\times} \times[n]\right)\right|}$ for all $y \in B_{n}$. Let $\sigma \in S_{n}$, and assume that $\sigma$ is the product of pairwise disjoint $n_{j}$-cycles $\sigma_{j}$ for $j=1,2, \ldots, r$ with $\sum_{j} n_{j}=n$. Let $\left(x_{1}, \ldots, x_{n}\right) \in V_{n}$, and set $y=\left(x_{1}, \ldots, x_{n}\right) \sigma$. For each $j \in\{1,2, \ldots, r\}$, let $I_{j}$ be the minimal subset of $[n]$ with $\sigma_{j} \in S_{I_{j}}$, and set

$$
y_{j}=\left(x_{1}^{(j)}, x_{2}^{(j)}, \ldots, x_{n}^{(j)}\right) \sigma_{j} \quad \text { with } \quad x_{i}^{(j)}=\left\{\begin{array}{cl}
x_{i} & \text { if } i \in I_{j}, \\
1 & \text { otherwise }
\end{array}\right.
$$


Obviously, $y=\prod_{j=1}^{r} y_{j}$. We now set $s=\sharp\left\{j \in\{1,2, \ldots, r\} \mid \prod_{i=1}^{n} x_{i}^{(j)}=1\right\}$, so that $\left|\langle y\rangle \backslash\left(\mathbb{Z}^{\times} \times[n]\right)\right|=r+s$. Hence it turns out that

$$
\kappa_{n}(y)=\prod_{i=1}^{n} x_{i}=\prod_{j=1}^{r} \prod_{i=1}^{n} x_{i}^{(j)}=(-1)^{r-s}=(-1)^{\left|\langle y\rangle \backslash\left(\mathbb{Z}^{\times} \times[n]\right)\right|} .
$$

Consequently, we obtain $\kappa_{n}=\operatorname{char}\left(\widetilde{\Lambda}_{P\left(\mathbb{Z}^{\times} \times[n]\right)}\right)$, completing the proof.

The following lemma, which is a basic fact for the left $B_{n^{-s e t}} \mathbb{Z}^{\times} \times[n]$, is crucial.

Lemma 5.7 Let $\left\{M_{1}, \ldots, M_{i}\right\}, i$ a positive integer, be a partition of $\mathbb{Z}^{\times} \times[n]$ into nonempty subsets, and view them as elements of the $B_{n}$-poset $P\left(\mathbb{Z}^{\times} \times[n]\right)$. If each $M_{j}$ for $j=1,2, \ldots, i$ does not include both $(1, q)$ and $(-1, q)$ for any $q \in[n]$, then there exists an element $\lambda$ of $\mathcal{P}(n)$ such that the intersection of stabilizers of $M_{j}$ in $B_{n}$ for $j=1,2, \ldots, i$ is a conjugate of $S_{\lambda}$.

Proof. There is a partition $\left\{N_{1}, \ldots, N_{k}\right\}, k$ a positive integer, of $[n]$ into nonempty subsets such that each $M_{j}$ for $j=1,2, \ldots, i$ consists of either $(1, q)$ or $(-1, q)$, but not both, for each $q \in N_{\ell_{1}} \dot{\cup} \cdots \dot{\cup} N_{\ell_{r}}$ with $\left\{N_{\ell_{1}}, \ldots, N_{\ell_{r}}\right\} \subset\left\{N_{1}, \ldots, N_{k}\right\}$. Let $\widehat{\mathcal{P}}(n)$ be the set of all cycle types to which such partitions $\left\{N_{1}, \ldots, N_{k}\right\}$ of $[n]$ into nonempty subsets correspond, and take the maximal element $\mu$ of $\widehat{\mathcal{P}}(n)$ with respect to the partially order $\leq$ on $\mathcal{P}(n)$ given in the proof of Lemma 5.5. Let $\left\{N_{1}, \ldots, N_{k}\right\}$ be a partition of $[n]$ into nonempty subsets corresponding to $\mu$ which satisfy the above condition. We set $J=N_{\ell}$, where $\ell$ is an arbitrary integer with $1 \leq \ell \leq k$. There exists a unique subset $Q$ of $J$ such that

$$
J^{+}:=\{(1, q) \mid q \in Q\} \dot{\cup}\{(-1, q) \mid q \in J-Q\} \subset M_{j_{1}}
$$

and

$$
J^{-}:=\{(1, q) \mid q \in J-Q\} \dot{\cup}\{(-1, q) \mid q \in Q\} \subset M_{j_{2}}
$$

for some integers $j_{1}$ and $j_{2}$ with $1 \leq j_{1} \neq j_{2} \leq i$. Let $g=\left(x_{1}, \ldots, x_{n}\right) \in L_{Q}$, and suppose that $x_{q}=-1$ for all $q \in Q$. Then the stabilizer of $J^{+}$in $B_{n}$ is $g\left(L_{\bar{J}} S_{J} S_{\bar{J}}\right)$, and so is that of $J^{-}$in $B_{n}$. Observe now that the intersection of stabilizers of $M_{j}$ for $j=1,2, \ldots, i$ in $B_{n}$ coincides with the intersection of such subgroups of $B_{n}$. Hence the assertion is a consequence of Lemma 5.1. This completes the proof.

Identifying $(-1, q)$ with $n+q \in[2 n]$ for all $q \in[n]$, we may consider $S_{2 n}$ to be the symmetric group on $\mathbb{Z}^{\times} \times[n]$. In particular, $B_{n}$ is viewed as a subgroup of $S_{2 n}$.

Lemma 5.8 Let $\lambda \in \mathcal{P}(2 n)$. Then $B_{n} \cap{ }^{\sigma} S_{\lambda} \in \overline{\mathcal{U}}_{n}$ for all $\sigma \in S_{2 n}$, and

$$
\left[\operatorname{res}_{B_{n}}^{S_{2 n}}\left(S_{2 n} / S_{\lambda}\right)\right]=\sum_{\sigma \in \overline{B_{n} \backslash S_{2 n} / S_{\lambda}}}\left[B_{n} /\left(B_{n} \cap{ }^{\sigma} S_{\lambda}\right)\right] \in \Omega\left(B_{n}, \mathcal{U}_{n}\right),
$$

where $\operatorname{res}_{B_{n}}^{S_{2 n}}$ indicates restriction of operators from $S_{2 n}$ to $B_{n}$ and $\overline{B_{n} \backslash S_{2 n} / S_{\lambda}}$ is a complete set of representatives of double cosets $B_{n} \sigma S_{\lambda}, \sigma \in S_{2 n}$, in $S_{2 n}$. 
Proof. Let $\sigma \in S_{2 n}$. By Lemma 5.7, $B_{n} \cap{ }^{\sigma} S_{\lambda}={ }^{g \tau}\left(L_{J} S_{\mu_{J} \mu_{\bar{J}}}\right)$ for some $J \subset[n]$, $g \in L_{\bar{J}}, \tau \in S_{J} S_{\bar{J}}$, and $\left(\mu_{J}, \mu_{\bar{J}}\right) \in \mathcal{P}(J, \bar{J})$. Hence $B_{n} \cap{ }^{\sigma} S_{\lambda} \in \overline{\mathcal{U}}_{n}$. The second assertion follows from $[4$, (80.27) Subgroup Theorem]. This completes the proof.

There is a formula of the reduced Lefschetz invariant $\widetilde{\Lambda}_{P\left(\mathbb{Z}^{\times} \times[n]\right)}$ (cf. Eq.(6)) which is implicit in the proof of a conclusion from the proceeding facts:

Theorem 5.9 Define three elements $\alpha_{n}, \beta_{n}$, and $\gamma_{n}$ of $\Omega\left(B_{n}, \mathcal{U}_{n}\right)$ by

$$
\begin{aligned}
\alpha_{n} & =\sum_{i=0}^{n} \sum_{\lambda=\left(1^{m_{1}}, \ldots, i^{m_{i}}\right) \in \mathcal{P}(i)}(-1)^{m_{1}+\cdots+m_{i}+n} \frac{\left(m_{1}+\cdots+m_{i}\right) !}{m_{1} ! \cdots m_{i} !}\left[B_{n} /\left(S_{\lambda} B_{n-i}\right)\right], \\
\beta_{n} & =(-1)^{n} \widetilde{\Lambda}_{P([n])}, \quad \text { and } \quad \gamma_{n}=\widetilde{\Lambda}_{P\left(\mathbb{Z}^{\times} \times[n]\right)} .
\end{aligned}
$$

Then $\varepsilon_{n}=\operatorname{char}\left(\alpha_{n}\right), \rho_{n}=\operatorname{char}\left(\beta_{n}\right), \kappa_{n}=\operatorname{char}\left(\gamma_{n}\right)$, and $\alpha_{n}=(-1)^{n} \widetilde{\Lambda}_{P\left([n] \dot{\cup}\left(\mathbb{Z}^{\times} \times[n]\right)\right)}$. Moreover, $\Omega\left(B_{n}, \mathcal{E}_{n}\right)^{\times}=\left\langle\alpha_{n},-1\right\rangle, \Omega\left(B_{n}, \mathcal{U}_{n}\right)^{\times}=\left\langle\beta_{n}, \gamma_{n},-1\right\rangle$, and $\alpha_{n}=\beta_{n} \gamma_{n}$.

Proof. By Eq.(5), $\varepsilon_{n}=\operatorname{char}\left(\alpha_{n}\right)$. Obviously, $\alpha_{n} \in \Omega\left(B_{n}, \mathcal{E}_{n}\right)$. Since $\alpha_{n} \neq 1,-1$, it follows from Proposition 5.4 that $\Omega\left(B_{n}, \mathcal{E}_{n}\right)^{\times}$is generated by $\alpha_{n}$ and -1 . By Proposition 4.3 and Lemma 5.6, we have $\rho_{n}=\operatorname{char}\left(\beta_{n}\right), \beta_{n} \in \Omega\left(B_{n}, \mathcal{U}_{n}\right)$, and $\kappa_{n}=\operatorname{char}\left(\gamma_{n}\right)$. The reduced Lefschetz invariant $\widetilde{\Lambda}_{P([2 n])}$ of the left $S_{2 n}$-set $[2 n]$ is an element of $\Omega\left(S_{2 n}, \mathcal{Y}_{2 n}\right)$ (cf. [9, $\left.\S 4\right]$ ); for its description, see Eq.(3). We may identify $\widetilde{\Lambda}_{P\left(\mathbb{Z}^{\times} \times[n]\right)}$ with $\operatorname{res}_{B_{n}}^{S_{2 n}}\left(\widetilde{\Lambda}_{P([2 n])}\right)$ which is the element of $\Omega\left(B_{n}\right)$ obtained by restriction of operators on $S_{2 n}$-sets appearing in the components of $\widetilde{\Lambda}_{P([2 n])}$ from $S_{2 n}$ to $B_{n}$. By Lemma 5.8, $\operatorname{res}_{B_{n}}^{S_{2 n}}\left(\widetilde{\Lambda}_{P([2 n])}\right) \in \Omega\left(B_{n}, \mathcal{U}_{n}\right)$, and thus $\widetilde{\Lambda}_{P\left(\mathbb{Z}^{\times} \times[n]\right)} \in \Omega\left(B_{n}, \mathcal{U}_{n}\right)$. Moreover, it follows from Lemma 4.2 and Corollary 5.3 that $\Omega\left(B_{n}, \mathcal{U}_{n}\right)^{\times}$is generated by $\beta_{n}, \gamma_{n}$, and -1 . Also, $\alpha_{n}=\beta_{n} \gamma_{n}$, because $\varepsilon_{n}=\rho_{n} \kappa_{n}$. By Proposition 2.3, it turns out that $\alpha_{n}=(-1)^{n} \widetilde{\Lambda}_{P\left([n] \dot{\cup}\left(\mathbb{Z}^{\times} \times[n]\right)\right)}$. This completes the proof.

Since $\widetilde{\Lambda}_{P\left(\mathbb{Z}^{\times} \times[n]\right)}=\operatorname{res}_{B_{n}}^{S_{2 n}}\left(\widetilde{\Lambda}_{P([2 n])}\right)$, it follows from Eq.(3) and Lemma 5.8 that

$$
\begin{aligned}
& \widetilde{\Lambda}_{P\left(\mathbb{Z}^{\times} \times[n]\right)}=\sum_{\lambda=\left(1^{m_{1}}, \ldots,(2 n)^{m_{2 n}}\right) \in \mathcal{P}(2 n)} \sum_{\sigma \in \overline{B_{n} \backslash S_{2 n} / S_{\lambda}}}(-1)^{m_{1}+\cdots+m_{2 n}} \\
& \times \frac{\left(m_{1}+\cdots+m_{2 n}\right) !}{m_{1} ! \cdots m_{2 n} !}\left[B_{n} /\left(B_{n} \cap{ }^{\sigma} S_{\lambda}\right)\right] .
\end{aligned}
$$

We close this section with a character theoretical explanation of the formula of $\kappa_{n}$ obtained by Eq.(6). For each $\mathbb{C}$-character $\chi$ of $G$, let $\left.\chi\right|_{H}$ with $H \leq G$ denote the $\mathbb{C}$-character obtained by restriction of $\chi$ from $G$ to $H$.

Lemma 5.10 Let $\mathbf{M}: G \rightarrow G L_{n}(\mathbb{C})$ be a $\mathbb{C}$-representation of $G$ affording a real valued character $\chi$ of $G$. Then for any $g \in G$,

$$
\operatorname{det} \mathbf{M}(g)=(-1)^{n-\left\langle\left.\chi\right|_{\langle g\rangle}, 1\langle g\rangle\right.},
$$

where $\left\langle\left.\chi\right|_{\langle g\rangle}, 1_{\langle g\rangle}\right\rangle$ is the inner product of $\left.\chi\right|_{\langle g\rangle}$ and $1_{\langle g\rangle}$. 
Proof. See the later part of the proof of [14, Theorem A].

There is a representation $\mathbf{M}_{n}: S_{n} \rightarrow G L_{n}(\mathbb{C})$ given by

$$
\sigma \mapsto\left(\delta_{\sigma^{-1}(i) j}\right)_{1 \leq i, j \leq n}, \quad \delta \text { the Kronecker delta, }
$$

which affords the permutation character $\pi_{[n]}: S_{n} \rightarrow \mathbb{C}$. Obviously, the sign character $\operatorname{sgn}_{n}: S_{n} \rightarrow \mathbb{C}$ coincides with the linear $\mathbb{C}$-character $\operatorname{det} \mathbf{M}_{n}: S_{n} \rightarrow \mathbb{C}$ given by

$$
\sigma \mapsto \operatorname{det} \mathbf{M}_{n}(\sigma)
$$

for all $\sigma \in S_{n}$. Recall that $B_{n}$ is viewed as a subgroup of $S_{2 n}$. By Lemma 5.10,

$$
\operatorname{det} \mathbf{M}_{2 n}(\sigma)=(-1)^{\left\langle\pi_{[2 n]}\right|\langle\sigma\rangle, 1\langle\sigma\rangle}{ }_{\langle\sigma}=(-1)^{|\langle\sigma\rangle \backslash[2 n]|}
$$

for all $\sigma \in S_{2 n}$ (see also [9, Lemma 3.3]). This, combined with Proposition 2.3 and Lemma 5.6, shows that the linear $\mathbb{C}$-character $\left.\operatorname{det} \mathbf{M}_{2 n}\right|_{B_{n}}: B_{n} \rightarrow \mathbb{C}$ coincides with $\kappa_{n}: B_{n} \rightarrow \mathbb{C}$. Consequently, we have $\kappa_{n}=\left.\operatorname{sgn}_{2 n}\right|_{B_{n}}$. Hence it follows from Eq.(4) and Lemma 5.8 (see also [4, (10.13) Subgroup Theorem]) that

$$
\kappa_{n}=\sum_{\lambda=\left(1^{m_{1}}, \ldots,(2 n)^{m_{2 n}}\right) \in \mathcal{P}(2 n)} \sum_{\sigma \in \overline{B_{n} \backslash S_{2 n} / S_{\lambda}}}(-1)^{m_{1}+\cdots+m_{2 n}} \frac{\left(m_{1}+\cdots+m_{2 n}\right) !}{m_{1} ! \cdots m_{2 n} !} 1_{B_{n} \cap \sigma_{S_{\lambda}}}^{B_{n}}
$$

and $B_{n} \cap{ }^{\sigma} S_{\lambda} \in \overline{\mathcal{U}}_{n}$ for all $\lambda \in \mathcal{P}(2 n)$ and $\sigma \in S_{2 n}$.

\section{The parabolic Burnside rings of even-signed permutation groups}

We set $D_{n}=\operatorname{ker} \kappa_{n}$ and call it the even-signed permutation group on $[n]$. Obviously, $D_{n}=K_{n} S_{n}$, where $K_{n}=\operatorname{ker} \vartheta_{n}$. Suppose that $[i] \subset[n]$ and $\lambda \in \mathcal{P}(i)$. We set $S_{\lambda} D_{n-i}=\left(K_{n} \cap L_{\bar{i}}\right) S_{\lambda} S_{\bar{i}}$ and set $t=(0,0, \ldots, 1) \in V_{n}$. Observe that

$$
\left[\operatorname{res}_{D_{n}}^{B_{n}}\left(B_{n} /\left(S_{\lambda} B_{n-i}\right)\right)\right]= \begin{cases}{\left[D_{n} /\left(S_{\lambda} D_{n-i}\right)\right]} & \text { if } 0 \leq i \leq n-1, \\ {\left[D_{n} / S_{\lambda}\right]+\left[D_{n} /{ }^{t} S_{\lambda}\right]} & \text { if } i=n\end{cases}
$$

by $[4,(80.27)$ Subgroup Theorem], which are contained in the parabolic Burnside ring $\mathcal{P} \mathcal{B}\left(D_{n}\right)$ (cf. $[6,2.3 .11]$ ). We define a map $\operatorname{res}_{D_{n}}^{B_{n}}: \mathcal{P} \mathcal{B}\left(B_{n}\right) \rightarrow \mathcal{P B}\left(D_{n}\right)$ by

$$
\left[B_{n} /\left(S_{\lambda} B_{n-i}\right)\right] \mapsto\left[\operatorname{res}_{D_{n}}^{B_{n}}\left(B_{n} /\left(S_{\lambda} B_{n-i}\right)\right)\right]
$$

for all $[i] \subset[n]$ and $\lambda \in \mathcal{P}(i)$. Set $\alpha_{n}^{\prime}=\operatorname{res}_{D_{n}}^{B_{n}}\left(\alpha_{n}\right)$ (see Theorem 5.9). Then

$$
\begin{aligned}
& \alpha_{n}^{\prime}=\sum_{i=0}^{n-1} \sum_{\lambda=\left(1^{m_{1}}, \ldots, i^{m_{i}}\right) \in \mathcal{P}(i)}(-1)^{m_{1}+\cdots+m_{i}+n} \frac{\left(m_{1}+\cdots+m_{i}\right) !}{m_{1} ! \cdots m_{i} !}\left[D_{n} /\left(S_{\lambda} D_{n-i}\right)\right] \\
& +\sum_{\lambda=\left(1^{m_{1}}, \ldots, n^{m_{n}}\right) \in \mathcal{P}(n)}(-1)^{m_{1}+\cdots+m_{n}+n} \frac{\left(m_{1}+\cdots+m_{n}\right) !}{m_{1} ! \cdots m_{n} !}\left(\left[D_{n} / S_{\lambda}\right]+\left[D_{n} /{ }^{t} S_{\lambda}\right]\right) .
\end{aligned}
$$


Proposition 6.1 $\mathcal{P} \mathcal{B}\left(D_{n}\right)^{\times}=\left\langle\alpha_{n}^{\prime},-1\right\rangle$.

Proof. By the proof of [1, Theorem 4.5], there is an injection from $\mathcal{P B}\left(D_{n}\right)^{\times}$to $R\left(D_{n}\right)^{\times}$inherited from the ring homomorphism char $: \Omega\left(D_{n}\right) \rightarrow R\left(D_{n}\right)$. The sign character $\left.\varepsilon_{n}\right|_{D_{n}}: D_{n} \rightarrow \mathbb{C}$ is the only nontrivial $\mathbb{C}$-character of $D_{n}$ and $\mathbb{Q}$ is a splitting field for $D_{n}$ (cf. $[6, \S 5.6]$ ). This, combined with [13, Corollary 1.2 and Lemma 2.1], shows that $R\left(D_{n}\right)^{\times}$is isomorphic to the four group. Moreover, by [4, (10.13) Subgroup Theorem] and Eq.(5), we have $\left.\varepsilon_{n}\right|_{D_{n}}=\operatorname{char}\left(\alpha_{n}^{\prime}\right)$. Consequently, $\mathcal{P B}\left(D_{n}\right)^{\times}$is generated by $\alpha_{n}^{\prime}$ and -1 . This completes the proof.

Remark 6.2 Let $(W, S)$ be a Coxeter system of type $E_{6}, E_{7}$, or $E_{8}$. Then every character of $W$ is rational-valued (cf. [6, 5.3.6]). Moreover, there are exactly two linear $\mathbb{C}$-characters of $W$ (cf. [6, pp. 413-416]). Hence $R(W)^{\times}$is isomorphic to the four group and $\mathcal{P B}(W)^{\times}$is isomorphic to a subgroup of $R(W)^{\times}$(see the proof of Proposition 6.1). Thus it follows from [4, (66.29) Corollary] that $\mathcal{P} \mathcal{B}(W)^{\times}$is of order 4 and is generated by $\sum_{J \subset S}(-1)^{|J|}\left[W / W_{J}\right]$ and -1 , where $W_{J}=\langle s \mid s \in J\rangle$.

\section{REFERENCES}

[1] F. Bergeron, N. Bergeron, R. B. Howlett, and D. E. Taylor, A decomposition of the descent algebra of a finite Coxeter group, J. Algebraic Combin. 1 (1992), 23-44.

[2] R. Boltje and B. Külshammer, Canonical Brauer induction and symmetric groups, Boll. Unione Mat. Ital. Sez. B Artic. Ric. Mat. (8) 8 (2005), 453-460.

[3] S. Bouc, Burnside rings, Handbook of algebra, Vol. 2, 739-804, North-Holland, Amsterdam, 2000.

[4] C. W. Curtis and I. Reiner, Methods of Representation Theory, Vol. I, II, Wiley-Interscience, New York, 1981, 1987.

[5] A. Dress, Operations in representation rings, in "Representation theory of finite groups and related topics," (Madison, Wis., 1970), 39-45, Proc. Sympos. Pure Math., Vol. XXI, Amer. Math. Soc., Providence, R.I., 1971.

[6] M. Geck and G. Pfeiffer, Characters of finite Coxeter groups and IwahoriHecke algebras, London Mathematical Society Monographs, New Series, 21, The Clarendon Press, Oxford University Press, New York, 2000.

[7] L. Geissinger and D. Kinch, Representations of the hyperoctahedral groups, J. Algebra, 53 (1978), 1-20.

[8] B. Huppert, Character Theory of Finite Groups, de Gruyter Expositions in Mathematics, 25, Walter de Gruyter, Berlin, 1998. 
[9] F. Oda, Y. Takegahara, and T. Yoshida, The units of a partial Burnside ring relative to the Young subgroups of a symmetric group, J. Algebra, 460 (2016), 370-379.

[10] R. P. Stanley, Enumerative Combinatorics, Vol. I, Cambridge University Press, Cambridge, 1997.

[11] J. Thévenaz, Permutation representations arising from simplicial complexes, J. Combin. Theory Ser. A 46 (1987), 121-155.

[12] E. Yalçın, An induction theorem for the unit groups of Burnside rings of 2groups, J. Algebra 289 (2005), 105-127.

[13] K. Yamauchi, The construction of units of infinite order in the character ring of a finite group, Yokohama Math. J. 51 (2005), 89-97.

[14] T. Yoshida, On the unit groups of Burnside rings, J. Math. Soc. Japan 42 (1990), 31-64.

[15] T. Yoshida, The generalized Burnside ring of a finite group, Hokkaido Math. J. 19 (1990), 509-574. 